\title{
First observations and suppression of multipass, multibunch beam breakup in the Jefferson Laboratory free electron laser upgrade
}

\author{
Christopher D. Tennant, Kevin B. Beard, David R. Douglas, Kevin C. Jordan, Lia Merminga, and Eduard G. Pozdeyev \\ Thomas Jefferson National Accelerator Facility, Newport News, Virginia 23606, USA \\ Todd I. Smith \\ W. W. Hansen Experimental Physics Laboratory, Stanford, California 94305, USA
}

(Received 17 January 2005; published 29 July 2005)

\begin{abstract}
The multipass, multibunch beam breakup (BBU) instability imposes a potentially severe limitation to the average current that can be accelerated in an energy-recovery linac. Simulation results for Jefferson Lab's free electron laser (FEL) upgrade driver are presented which predict the occurrence of BBU below the nominal operating current of the machine. In agreement with simulation, BBU was observed and preliminary measurements to identify the higher-order mode causing the instability are shown. In addition, measurements performed to experimentally determine the threshold current are described. Using a newly developed two-dimensional BBU simulation code, we study the effect of optical suppression techniques, first proposed in 1980 [R. E. Rand and T. I. Smith, Part. Accel. 11, 1 (1980)], on the threshold current of the FEL. Specifically we consider the effect of (1) reflecting the betatron planes about an axis that is at $45^{\circ}$ between the vertical and horizontal axes and (2) rotating the betatron planes by $90^{\circ}$. In two-pass recirculators, a $90^{\circ}$ rotation can be effective at increasing the threshold current for BBU. The successful installation of a five skew-quadrupole reflector in the backleg of the FEL has been shown to be effective at suppressing the instability and comments on preliminary operational experience will be given.
\end{abstract}

DOI: 10.1103/PhysRevSTAB.8.074403

PACS numbers: 29.27.Bd

\section{INTRODUCTION}

Beam breakup (BBU) occurs in all accelerators at sufficiently high currents. In recirculating accelerators, in particular, energy-recovery linacs, the maximum current may be limited by multipass, multibunch BBU, a form that occurs when the electron beam interacts with the higherorder modes (HOMs) of an accelerating cavity on the accelerating pass and then again on higher passes. This effect is of particular concern in the design of modern high average current energy-recovery accelerators utilizing superconducting technology due to relatively high quality factors of the HOMs.

Following simulations which indicated BBU would occur below the nominal $10 \mathrm{~mA}$ operating current of the Jefferson Lab $10 \mathrm{~kW}$ free electron laser (FEL) upgrade driver, BBU was observed at $3 \mathrm{~mA}$ of average beam current in May 2004. In this paper we present experimental measurements to identify the HOM responsible for the breakup and measurements used to determine the threshold current for stability. With the knowledge that beam breakup imposes a real limitation to machine operation, the focus turned to finding a means of suppressing the instability.

The majority of this paper describes how the BBU threshold can be brought to a sufficiently large value by modifying the recirculation transfer matrix from a cavity back to itself. Applying conventional methods of suppressing beam instabilities - such as beam-based feedback and HOM damping techniques — are discussed elsewhere [1].
In this paper we specifically consider two beam optical schemes for BBU suppression first discussed in [2]. The first requires modifying the single turn recirculation matrix from the unstable cavity back to itself to produce a reflection of the betatron planes about an axis at $45^{\circ}$ between the horizontal and vertical axes which is effective at suppressing BBU for specific HOM orientations. The second method involves modifying the recirculation matrix to produce a $90^{\circ}$ rotation of the betatron planes. It is shown that for a given distribution of HOM orientations, this method is more efficient at suppressing BBU than a reflection. In this paper we have investigated applying reflecting optics and rotating optics to enhance the stability of the Jefferson Lab FEL upgrade driver. Care should be taken in applying these conclusions to larger scale accelerators where HOMs from many cavities may be responsible for the instability.

The driver is an energy-recovery based linear accelerator used to condition an electron beam for high power lasing. Electrons are injected at $10 \mathrm{MeV}$ and are accelerated to $145 \mathrm{MeV}$ through three cryomodules (each containing 8 superconducting niobium cavities). The beam is transported to a wiggler where up to $10 \mathrm{~kW}$ of laser power is generated [3]. The spent electron beam is recirculated and phased in such a way that the beam is decelerated through the linac region on the second pass. Upon exiting the linac, the $10 \mathrm{MeV}$ energy recovered beam is extracted to a dump (see Fig. 1 for a layout of the FEL). 


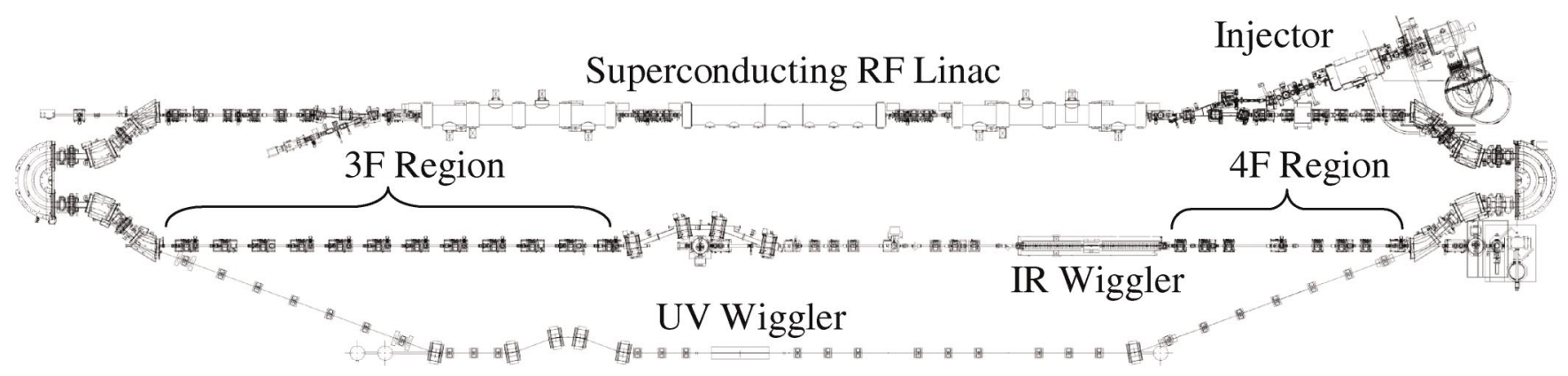

FIG. 1. Layout of the Jefferson Lab FEL $10 \mathrm{~kW}$ upgrade (the UV transport line has not yet been commissioned).

\section{EFFECTS OF ARBITRARY MODE POLARIZATION AND GENERALIZED TRANSPORT ON BBU}

A beam bunch will excite dipole HOMs in a cavity if it passes through the cavity off axis. The magnetic field of the excited mode then acts to deflect the following bunches. The kick produced by the mode is translated into a transverse displacement at the cavity after recirculation. Thus, the recirculated beam constitutes a feedback which can cause the voltage of the HOMs to grow. We consider a cavity containing a single HOM and a single beam recirculation. However, instead of limiting the HOM orientation to either $0^{\circ}$ or $90^{\circ}$, we allow for an arbitrary orientation angle, $\alpha$, with respect to the horizontal axis. We also allow for a full $4 \times 4$ recirculation matrix (i.e., the off-diagonal $2 \times 2$ matrices need not be zero). In calculating the threshold current, it is assumed that the induced HOM voltage on the second pass is small and can be treated as a perturbation. While a thorough derivation is given in [4,5], here we only give the result that the threshold current is approximately given by the following expression:

$$
\begin{aligned}
I_{\text {th }} & =-\frac{2 p_{b} c}{e k M^{*}(R / Q) Q \sin \left(\omega T_{r}\right)} \\
M^{*} & \equiv M_{12} \cos ^{2} \alpha+\left(M_{14}+M_{32}\right) \sin \alpha \cos \alpha+M_{34} \sin ^{2} \alpha,
\end{aligned}
$$

where $p_{b}$ is the beam momentum at the cavity, $c$ is the speed of light, $e$ is the electron charge, $k$ is the wave number $(\omega / c)$ of the HOM, $(R / Q) Q$ is the shunt impedance of the $\mathrm{HOM}, T_{r}$ is the recirculation time, and the $M_{i j}$ are the elements of the recirculation transport matrix from the cavity back to itself (which can describe coupled transverse motion). A derivation of $M^{*}$ is given in the appendix. This equation is a good approximation only under the condition that $M^{*} \sin \left(\omega T_{r}\right)<0$. A more rigorous treatment of BBU in energy recovering linacs can be found in [6] which considers the one-dimensional case only. As will be evident shortly, the functional dependence on mode orientation leads to important consequences for BBU suppression techniques.

\section{BBU SIMULATION PREDICTIONS FOR THE FEL}

The simulation code MATBBU $[7,8]$ developed at Jefferson Laboratory was used to predict the threshold currents in the $10 \mathrm{~kW}$ FEL upgrade driver. Unlike its predecessor TDBBU [9,10], MATBBU solves for the threshold current analytically, making use of the dispersion relation that exists between the beam current and frequency. At sufficiently high current the beam motion becomes unstable which is marked by the complex frequency having a positive imaginary part. The point at which this frequency intersects the real axis of the complex current plane gives the threshold current [6]. By sweeping the frequency, MATBBU can locate these instabilities, specifying the frequency and current at which they occur. The threshold current for a given machine configuration is the lowest of these currents.

Modeling BBU requires information to fully characterize the HOMs of interest and also an accurate description of the machine optics. An HOM is characterized by its $R / Q$, frequency, loaded quality factor, and polarization. Of these parameters, only the polarization is not known for the HOMs of interest in the FEL. The frequency and $Q_{L}$ were measured directly from the cold cryomodule and the $R / Q$ was modeled by MAFIA [11]. Only worst case scenarios were simulated. That is, each HOM was modeled twice in the simulation - one with a polarization of 0 degrees and the other with a polarization of $90^{\circ}$.

Design optics for $145 \mathrm{MeV}$ beam energy operation were generated by the particle tracking code DIMAD [12] and used to characterize the recirculator. A feature of MATBBU (and TDBBU) is the option to explicitly define transfer matrices for each accelerating cavity. In this way cavity rf focusing can be included, which is known to have an appreciable effect-particularly at the front end of the linac where the energy of the injected beam is $10 \mathrm{MeV}$. These matrices were also generated by DIMAD.

The results of the MATBBU simulation are shown in Table I [13]. In the worst case scenario, the two most 
TABLE 1. Summary of the simulation results showing mode properties of those HOMs which are predicted to produce threshold currents below $10 \mathrm{~mA}$ with the FEL configured for $145 \mathrm{MeV}$ of beam energy operation. The expected threshold currents for $88 \mathrm{MeV}$ operation were determined by scaling the results at $145 \mathrm{MeV}$ by the ratio of the energies at the cavity containing each HOM.

\begin{tabular}{|c|c|c|c|c|c|}
\hline Frequency (MHz) & Loaded $Q$ & $(R / Q)(\Omega)$ & $\begin{array}{l}145 \mathrm{MeV} \\
\text { Threshold current (mA) }\end{array}$ & $\begin{array}{l}88 \mathrm{MeV} \\
\text { Threshold current (mA) }\end{array}$ & Orientation \\
\hline 2102.607 & $2.61 \times 10^{6}$ & 29.90 & 7.1 & 5.3 & $0^{\circ}$ \\
\hline 2104.683 & $1.94 \times 10^{6}$ & 29.90 & 7.9 & 4.8 & $0^{\circ}$ \\
\hline 2106.007 & $6.11 \times 10^{6}$ & 29.90 & 2.9 & 2.0 & $90^{\circ}$ \\
\hline 2114.156 & $5.21 \times 10^{6}$ & 28.80 & 3.7 & 2.1 & $0^{\circ}$ \\
\hline 2115.201 & $2.17 \times 10^{6}$ & 28.80 & 8.3 & 5.3 & $90^{\circ}$ \\
\hline 2116.055 & $3.06 \times 10^{6}$ & 28.80 & 5.0 & 2.5 & $0^{\circ}$ \\
\hline 2116.585 & $6.66 \times 10^{6}$ & 28.80 & 4.2 & 2.9 & $0^{\circ}$ \\
\hline
\end{tabular}

dangerous modes are the $\mathrm{TM}_{110} 3 \pi / 7$ mode in cavity 7 which produces a threshold current of $2.9 \mathrm{~mA}$ in the vertical plane and the $\mathrm{TM}_{110} 4 \pi / 7$ mode in cavity 4 which produces a threshold current of $3.7 \mathrm{~mA}$ in the horizontal plane. Additionally, there exist five other $\mathrm{TM}_{110}$ modes which have the potential to cause breakup below the nominal operating current of $10 \mathrm{~mA}$.

\section{EXPERIMENTAL MEASUREMENTS OF BBU}

Observations of BBU were precipitated by the installation of a new 7-cell cryomodule to increase the beam energy from $88 \mathrm{MeV}$ to the design energy of $145 \mathrm{MeV}$. Measurements to characterize the HOMs revealed several loaded $Q$ s on the order of $10^{6}$ which lead to the threshold currents given in Table I. During initial commissioning with the cryomodule installed, the cavities in the new cryomodule were run with modest gradients while the gradients of cavities in the remaining two cryomodules were lowered slightly so as to preserve the machine configuration for $88 \mathrm{MeV}$ operation. It was under these conditions that the first observations of BBU were made. Therefore the threshold current predictions for the design beam energy from simulations need to be scaled accordingly (e.g., by the ratio of the energies at the cavity containing the harmful HOM) before comparing with the experimental results (the machine configuration for $88 \mathrm{MeV}$ operation was generated by scaling the $145 \mathrm{MeV}$ design lattice with energy). This leads to the lowest threshold currents of $2.0 \mathrm{~mA}$ in the vertical plane $(2106.007 \mathrm{MHz})$ and $2.1 \mathrm{~mA}$ in the horizontal plane (2114.156 MHz).

Using Schottky diodes on each of the two HOM ports per cavity ( 8 cavities $\times 2$ ports $=16$ diodes total) we were able to monitor the total HOM power levels from each cavity in the 7-cell cryomodule. At the onset of BBU, the amplitude of the offending HOM grows exponentially and the beam is driven at the frequency of the HOM. Figure 2 shows the power and voltage together from the unstable cavity during a BBU induced machine trip. The $1 \mathrm{~V}$ measured from the Schottky diode in Fig. 2 corresponds to
$1.5 \mathrm{~W}$ of HOM power extracted from the coupler. We have observed up to $4 \mathrm{~W}$ of extracted HOM power during a beam breakup. If the oscilloscope used to measure the cavity voltage is fast enough and the signal sufficiently sampled, the frequency of the offending mode can be extracted by Fourier analysis. The FFT (fast-Fourier transform) of a voltage signal from the unstable cavity during a beam breakup is shown in the lower plot in Fig. 3 with the HOM frequency of $2114.156 \mathrm{MHz}$ clearly distinguishable. For comparison, the upper plot of Fig. 3 shows the expected FFT for a pure $2114.156 \mathrm{MHz}$ signal. The fact that we did not observe beam breakup as a result of the 2106.007 MHz HOM suggests that either (a) this mode is not oriented at $90^{\circ}$ (as simulated) and therefore does not pose a threat for causing BBU (see Table I) or that (b) the simulated optics do not accurately reflect those of the machine (at the time of these measurements we did not have the capability of accurately measuring and quantifying the machine optics). By the same token, identifying the

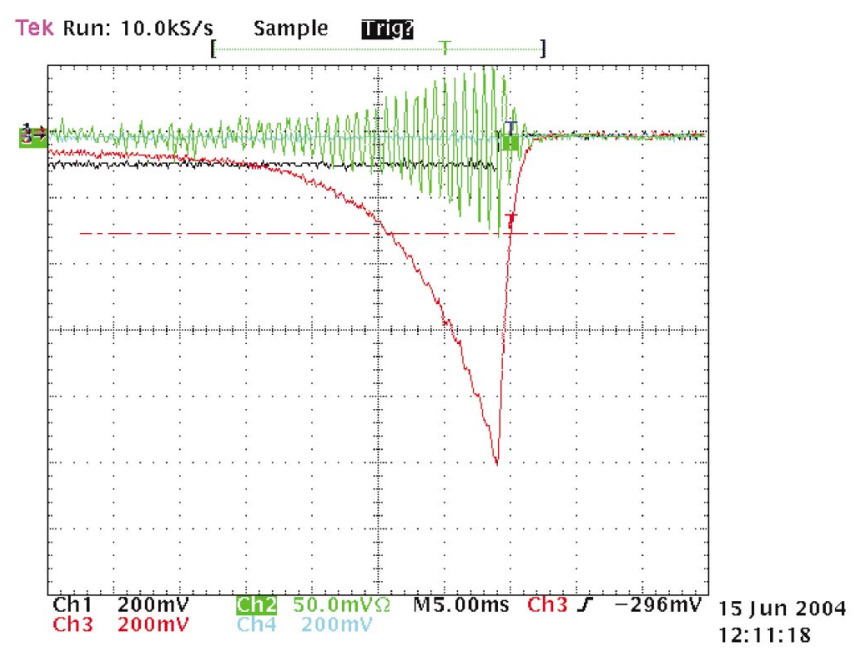

FIG. 2. (Color) Oscilloscope traces during a BBU induced machine trip. The green trace is the cavity HOM voltage and the red trace is the total HOM power as measured from a Schottky diode. The time scale is $5 \mathrm{msec} / \mathrm{div}$. 


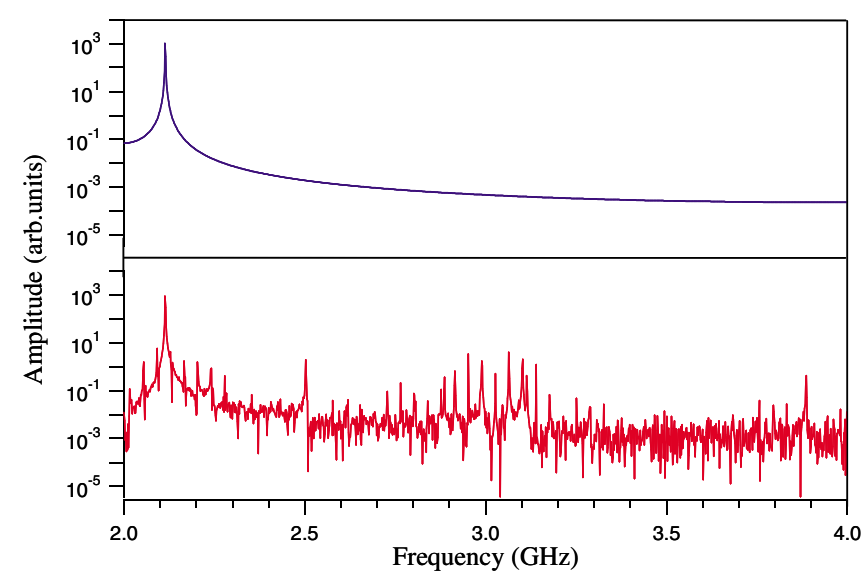

FIG. 3. (Color) FFT of a pure 2114.156 MHz signal (top) and FFT of the HOM voltage from the unstable cavity during beam breakup (bottom).

2114.156 MHz HOM as causing the breakup implies that it is oriented at very nearly $0^{\circ}$ in agreement with simulation. Measurements to verify these mode orientations are being planned.

With the flexibility in the beam bunching structures allowed by the injector, we can, in addition to observing BBU in continuous-wave (cw) mode operation, observe and measure BBU developing within a sufficiently long macropulse in pulsed mode operation. The evolution of the HOM voltage at currents above or below the threshold current is described by $[4,5]$

$$
V=V_{o} \exp \left(-\frac{\omega t}{2 Q_{L}} \frac{I_{\mathrm{th}}-I_{o}}{I_{\mathrm{th}}}\right)
$$

This is illustrated in Fig. 4 where the cavity voltage as measured through a Schottky diode is plotted against time for two $9 \mathrm{~ms}$ long macropulses of differing currents. The exponential growth represents the effective time constant

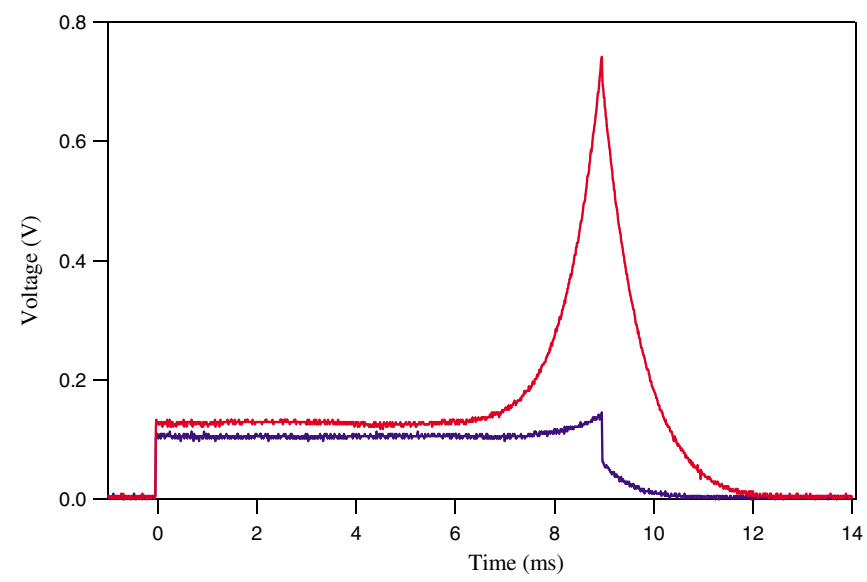

FIG. 4. (Color) HOM voltage measured from the unstable cavity for $9 \mathrm{~ms}$ long macropulses with $4.5 \mathrm{~mA}$ of average current (blue) and $5.0 \mathrm{~mA}$ of average current (red).

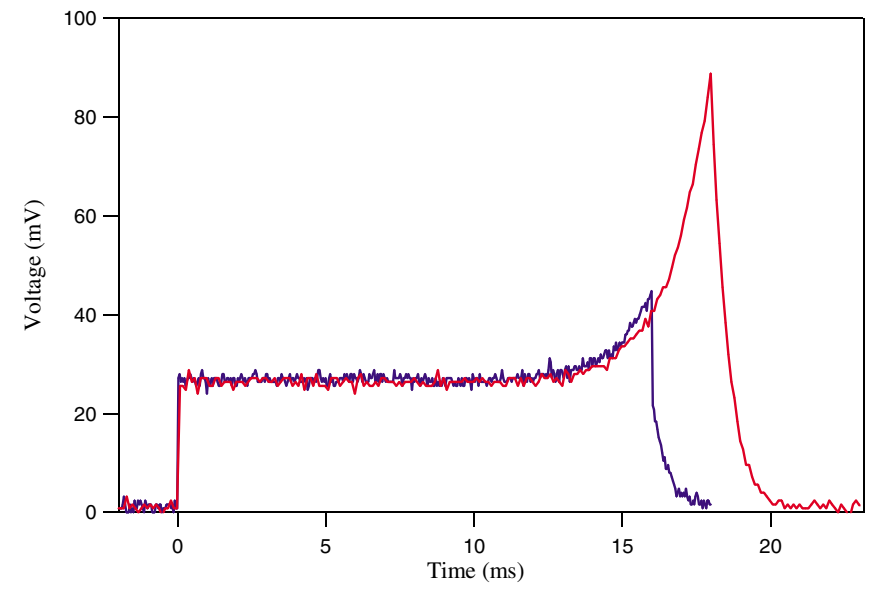

FIG. 5. (Color) HOM voltage measured from the unstable cavity for $16 \mathrm{~ms}$ (blue) and $18 \mathrm{~ms}$ (red) long macropulses with an average current of $3.5 \mathrm{~mA}$.

of the HOM-beam system. With the end of the macropulse, the exponential decay represents the natural damping time of the HOM causing the instability. The relationship between the two time constants is dependent on the average macropulse current at the time of the measurement and the threshold current. This relationship can be expressed equivalently in terms of the quality factors, using Eq. (2), as

$$
Q_{\mathrm{eff}}=\left(\frac{I_{\mathrm{th}}}{I_{\mathrm{th}}-I_{o}}\right) \cdot Q_{L},
$$

where $Q_{\text {eff }}$ is the effective quality factor of the HOM-beam system and $Q_{L}$ is the loaded quality factor of the HOM causing the instability.

The two traces shown in Fig. 4 each yield a threshold current of $(2.6 \pm 0.1) \mathrm{mA}$ using the method just described. The data in Fig. 5 illustrate the effect of keeping the macropulse current constant and changing the macropulse length. These measurements were performed several days after the data in Fig. 4, hence the machine setup is slightly different. Analyzing the traces yields a threshold current of $(2.4 \pm 0.1)$ and $(2.5 \pm 0.1) \mathrm{mA}$ from the 16 and $18 \mathrm{~ms}$ long macropulses, respectively. This is in excellent agreement with the direct measurement of the threshold current of $(2.3 \pm 0.2) \mathrm{mA}$ observed by operating in cw mode and noting the current at which the machine tripped off due to BBU induced beam losses. Furthermore, the loaded $Q$ of the HOM extracted from the data [see Eq. (3)] is within $15 \%$ agreement with previous rf measurements.

\section{BEAM OPTICAL METHODS FOR BBU SUPPRESSION}

We consider in detail two methods for beam optical control of BBU. The first method is a reflection of the betatron planes which is most effective at suppressing BBU when the HOMs are oriented at $0^{\circ}$ or $90^{\circ}$, while the second 
method utilizes a $90^{\circ}$ rotation of the betatron planes in which BBU is eliminated regardless of HOM orientation. The following discussion assumes the presence of a single HOM so that Eq. (1) can be applied. Although unrealistic, we consider this special case of a machine with single HOM so as to elucidate some features of a beam optical reflection and rotation. In the following section we will investigate the effects of many HOMs through simulations where it will be clear that mode coupling plays an important role.

\section{A. Reflection}

The transport matrix describing a reflection about a plane at $45^{\circ}$ to the horizontal or vertical axis takes the following form, where each element represents a $2 \times 2$ matrix

$$
\left(\begin{array}{cc}
0 & M \\
M & 0
\end{array}\right)
$$

The $2 \times 2$ sub-block transport matrix $M$ is the same for both exchanges ( $x$ to $y, y$ to $x$ ). Thus, such a reflector cleanly exchanges the horizontal and vertical phase spaces.

To see how a reflection can be effective in suppressing BBU, consider Eqs. (1) and (4). Since $M_{12}=M_{34}=0$, for a mode oriented at $0^{\circ}$ or $90^{\circ}$ the threshold current becomes infinite. However, if an HOM is rotated at an angle $\alpha$, not equal to $0^{\circ}$ or $90^{\circ}$, then the recirculated beam will not come back to the cavity with the angle $\left(\alpha+90^{\circ}\right)$ and its projection on the HOM will be nonzero. To get an infinite threshold for all HOM polarizations requires that $M_{32}=-M_{14}$.

\section{B. Rotation}

A more robust optical scheme which suppresses BBU regardless of $\mathrm{HOM}$ orientation is a $90^{\circ}$ rotation. The transport matrix describing a $90^{\circ}$ rotation takes the following form, where as before, each element represents a $2 \times 2$ matrix:

$$
\left(\begin{array}{cc}
0 & M \\
-M & 0
\end{array}\right)
$$

Consider now Eqs. (1) and (5). Since $M_{12}=M_{34}=0$ and $M_{32}=-M_{14}$, the threshold current is infinite and BBU is eliminated - independent of the mode orientation. A rotation implemented in a two-pass system effectively breaks the feedback loop formed between the beam and cavity HOM so there can be no exchange of energy. The idea is conceptually simple and is illustrated in Fig. 6. If on the first pass an offending mode imparts an angular deflection $\alpha$, to a bunch, then on the second pass (and after a $90^{\circ}$ rotation), the resultant displacement will be orthogonal to the deflection. Thus the bunch will be unable to couple energy to the mode that caused the deflection. In reality, however, the beam will couple with the orthogonal polarization of the mode. The effects of this mode coupling will

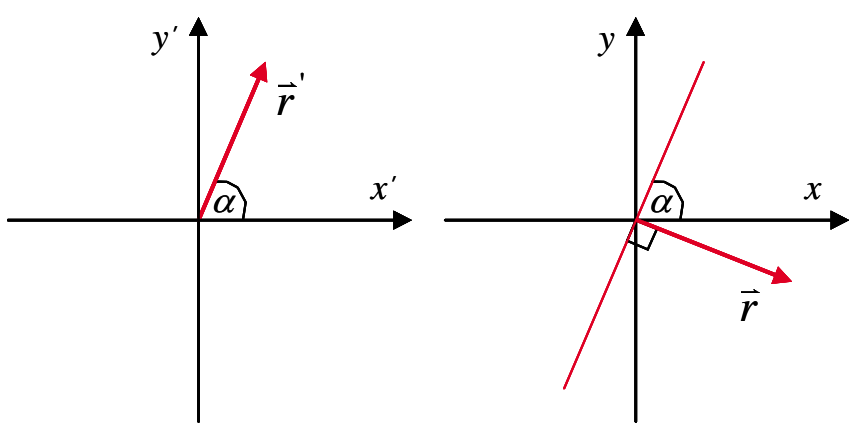

FIG. 6. (Color) Schematic illustrating the effect of a $90^{\circ}$ rotation of the betatron planes. A deflection on the first pass (left) is transformed to a displacement which is orthogonal to the deflection which caused it (right).

be evident in the next section where implementing a $90^{\circ}$ rotation in the presence of many HOMs still yields finite threshold currents.

\section{TWO-DIMENSIONAL BBU SIMULATION CODE AND RESULTS}

Simulations to study the effectiveness of the optical suppression schemes described above were performed using a newly developed BBU code [4]. The code was written in the standard ANSI $\mathrm{C}++$ language and the first version has been tested and benchmarked. The code works by tracking particles through a machine characterized by transfer matrices and follows the HOM energy buildups in accelerating cavities.

The code has been used to simulate beam breakup in several one-dimensional cases including the FEL upgrade driver. The results are in 3\% agreement with results simulated by the previous BBU simulation codes used at Jefferson Lab, TDBBU and MATBBU. Results for twodimensional simulations have been compared to the formula given in Eq. (1) and show excellent agreement with the theory [14]. In addition to handling two-dimensional transverse motion and arbitrarily oriented HOMs, the new code is faster than TDBBU and MATBBU by an order of magnitude or more depending on the particular problem.

To see the effectiveness of the two aforementioned methods for beam optical control of BBU, twodimensional simulations were performed. As in the previous simulations discussed, the simulation model uses the FEL design optics including the rf focusing effects and uses the measured data to describe the $28 \mathrm{HOMs}$ in each of the 8 cavities (frequencies and loaded $Q$ s). The only unknown is the polarization of each HOM. Thus two categories of simulations were performed. The first simulates HOMs with orientations of either $0^{\circ}$ or $90^{\circ}$ ("aligned modes") and the second simulates modes with orientations which are "skewed." To explore the effect of mode orientation further, as a subset of the "skewed modes" case, three distributions of HOM orientations were generated 
corresponding to a change from the "aligned modes" with standard deviations of $5^{\circ}, 15^{\circ}$, and $25^{\circ}$. For each of these HOM conditions, three different machine optics configurations were simulated: (1) nominal, uncoupled optics; (2) pseudoreflector optics [that is, optics characterized by a transfer matrix similar in form to Eq. (4) but with the offdiagonal matrices not equal to each other and which represents the best we can do operationally in the FEL in achieving a pure reflection]; (3) rotated optics. The results are summarized in Table II.

\section{A. Nominal optics}

With no suppression techniques applied, the threshold current for the case of "aligned modes" is just under $3 \mathrm{~mA}$. As the deviation of the mode orientations from $0^{\circ}$ and $90^{\circ}$ increases, the threshold current also increases consistent with Eq. (1). That is, because the optics is decoupled and the modes well separated in frequency, coupling between modes is negligible and the current for stability can be determined by the threshold current due to the worst individual mode using Eq. (1).

\section{B. Pseudoreflecting optics}

With the modes "aligned" the threshold current is increased from the nominal optics case by a factor of $\sim 20$. The point of interest however, is that with the mode orientations "skewed," the effectiveness of the pseudoreflection for increasing the threshold current rapidly diminishes. Thus, unless it is known that dangerous HOMs are oriented very nearly to $0^{\circ}$ or $90^{\circ}$, a reflection scheme may not adequately suppress BBU.

\section{Rotated optics}

For the case of "aligned modes" the threshold current is increased by a factor of $\sim 25$ which is similar to the effect of the pseudoreflection. Yet when the mode orientations become "skewed," a rotation becomes more effective at suppressing BBU than the pseudoreflection.

The objective of this study was to bring attention to the fact that HOM orientations can play an important role in both BBU and the choice of suppression techniques which had hitherto been overlooked. One of the salient conclusions is that for a given distribution of mode orientations, a rotation is more effective at increasing the threshold cur-

TABLE 2. Predicted threshold currents from two-dimensional BBU simulations where the standard deviation $(\sigma)$ represents the shift in mode orientation from $0^{\circ}$ and $90^{\circ}$.

\begin{tabular}{ccccc}
\hline \hline & $\sigma$ & Nominal & Pseudoreflection & Rotation \\
\hline Aligned & $0^{\circ}$ & $2.87 \mathrm{~mA}$ & $66.80 \mathrm{~mA}$ & $69.92 \mathrm{~mA}$ \\
Skewed & $5^{\circ}$ & $2.95 \mathrm{~mA}$ & $23.24 \mathrm{~mA}$ & $48.05 \mathrm{~mA}$ \\
& $15^{\circ}$ & $3.77 \mathrm{~mA}$ & $6.88 \mathrm{~mA}$ & $103.91 \mathrm{~mA}$ \\
& $25^{\circ}$ & $3.95 \mathrm{~mA}$ & $7.18 \mathrm{~mA}$ & $24.80 \mathrm{~mA}$ \\
\hline \hline
\end{tabular}

rent compared to the pseudoreflection. Keep in mind that these simulations were done for the Jefferson Laboratory FEL upgrade driver which is a relatively compact accelerator (433 ns recirculation time). For larger accelerators, especially with many cryomodules, using these beam optical schemes in the presence of many more HOMs could cause further destructive mode interference thus rendering these suppression techniques ineffective.

\section{IMPLEMENTATION OF BEAM OPTICAL ROTATION AND REFLECTION}

Having shown the relative merits of the aforementioned optical suppression schemes through simulations, we present several options for implementing them in the FEL upgrade recirculator.

\section{A. Reflection with skew quadrupoles}

A practical implementation of a reflector using skew quadrupoles has been noninvasively embedded in the $3 \mathrm{~F}$ region of the FEL upgrade driver (see Fig. 7) [15]. Operationally, normal quadrupoles upstream and downstream of the module are used as betatron matching telescopes. These allow transverse matching of the phase spaces across the reflector, so that the $3 \mathrm{~F}$ region, in its entirety, remains transparent to the rest of the machine. Apart from the exchange of horizontal and vertical emittance, the interchange of incident steering, and possible differences in phase advance, nothing is changed. While the skew quadrupoles produce a "local" reflection across the $3 \mathrm{~F}$ region, the transport matrix of interest for suppressing BBU describes the path from the unstable cavity back

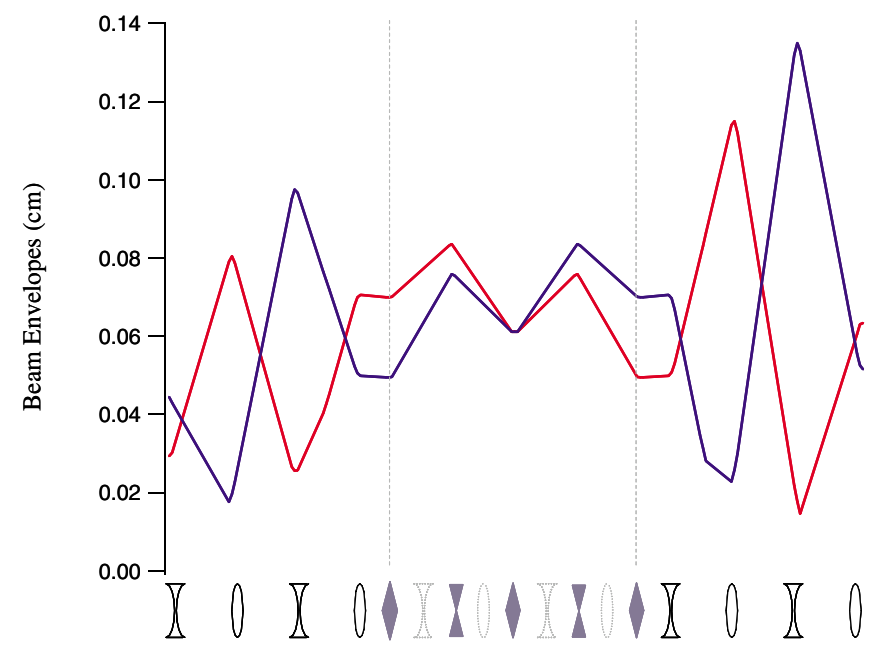

FIG. 7. (Color) Beam envelopes (horizontal in red and vertical in blue) for the $3 \mathrm{~F}$ region of the FEL with the five skew quadrupoles (blue) activated and illustrating the exchange of horizontal and vertical phase spaces. The central four normal quadrupoles (dotted) are deexcited during reflector operation and the upstream and downstream quadrupoles (black) are used as betatron matching telescopes. 
to itself. Thus, with nominal operating conditions, the effect of the embedded skew-quadrupole reflector is to produce a pseudoreflection; a matrix similar in form to Eq. (4) but with the off-diagonal matrices unequal.

\section{B. Rotation with a solenoid}

While utilizing a solenoid to produce a beam rotation seems like a natural choice, it is shown that the strong focusing incurred by its use makes it undesirable in low energy machines, and in high energy machines the large solenoid strength required makes it impractical. The $4 \times 4$ transport matrix for a solenoid which provides a $90^{\circ}$ rotation is given by

$$
\left(\begin{array}{cccc}
0 & 0 & 0 & 1 / K \\
0 & 0 & -K & 0 \\
0 & -1 / K & 0 & 0 \\
K & 0 & 0 & 0
\end{array}\right),
$$

where $K=B_{o} / 2 B \rho$ is the solenoid strength, $B_{o}$ is the field inside the solenoid, and $B \rho$ is the rigidity of the beam. For initial studies of the feasibility of beam optical suppression methods, an appropriate set of optics was generated for incorporating a solenoid in the FEL upgrade driver. Quadrupole triplets upstream and downstream of the solenoid are used to transversely match the optics in the $4 \mathrm{~F}$ region following the wiggler. For a solenoid length of $75 \mathrm{~cm}$, a solution of the matched betatron functions in the $4 \mathrm{~F}$ region is shown in Fig. 8 . For a $90^{\circ}$ rotation the required solenoid strength, in terms of its length, is given by $K=\pi / 2 l$. The $75 \mathrm{~cm}$ solenoid used in this exercise translates to a solenoid strength of $2.1 \mathrm{~m}^{-1}$ or a magnetic field of $20 \mathrm{kG}$. Although this solenoid strength is feasible for the $145 \mathrm{MeV}$ beam in the FEL, the strength required to

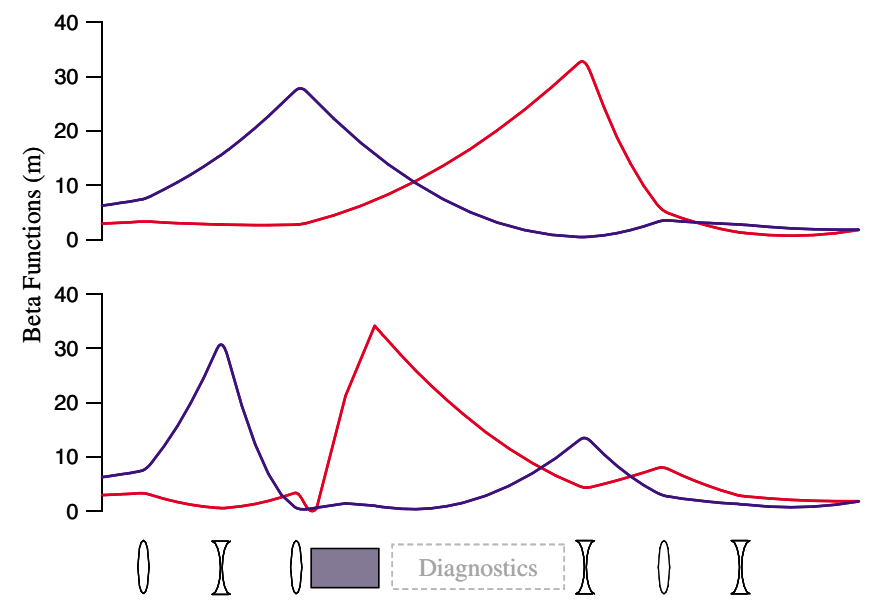

FIG. 8. (Color) Nominal betatron functions in the $4 \mathrm{~F}$ region of the FEL (top) and with a solenoid (blue) activated to produce a $90^{\circ}$ rotation of betatron planes with the upstream and downstream quadrupole triplets used for matching (bottom). Horizontal betatron functions are denoted in red and vertical in blue. rotate a beam of energy on the order of a $\mathrm{GeV}$ makes this method impractical. Another severe disadvantage of utilizing a solenoid is the undesirable strong focusing required to match the transverse optics (see Fig. 8).

\section{Rotation with skew quadrupoles}

An embedded local reflection in the recirculator can, in principle, be made to produce a pure rotation from the unstable cavity back to itself. The reflector described previously simply interchanges horizontal and vertical phase spaces, imaging each identically into the other. This will map a horizontal kick into the vertical plane, and a vertical kick to the horizontal plane, and thus is effective for suppressing BBU driven by modes with these specific polarizations. However, it will equally map a diagonal displacement and kick back into a similar diagonal displacement and kick, thus failing to suppress BBU driven by an HOM of arbitrary polarization.

The goal is to produce a single turn recirculation matrix from the unstable cavity back to itself of the form given in Eq. (5). In principle, without any additional hardware in the FEL, decreasing the unreflected vertical phase advance around the recirculator by a quarter-betatron wavelength and activating the reflector in the $3 \mathrm{~F}$ region results in a single turn matrix from the middle of the 7-cell cryomodule back to itself that describes a nearly perfect $90^{\circ}$ rotation [16]. Thus an arbitrarily oriented HOM-driven kick from a middle cavity in the cryomodule (which is precisely the location of the unstable cavity) returns not only out of plane but also at a node in position. We note that if the transport along the linac axis is cylindrically symmetric (i.e., no quadrupoles), the suppression holds for all cavities.

\section{PRELIMINARY OPERATIONAL EXPERIENCE}

In addition to the beam optical reflecting and rotating schemes, Rand and Smith also suggested using point-topoint focusing (i.e., $M_{12}=M_{34}=0$ ) at all points along the linac to suppress BBU [2]. This has the result that a kick on the first pass will not be translated to a displacement on the second pass. In general, this condition cannot be satisfied for all points along the linac, but it is possible to produce point-to-point focusing at the location of the most offending HOM. In fact, operational experience with the FEL upgrade has shown that by tuning the betatron phase advances and match, it was possible to vary the BBUdriven current limit from as low as $1 \mathrm{~mA}$ to over $5 \mathrm{~mA}$ (the usual operational current). However, such configurations did not always reproduce over the course of days due to irreproducibility in the transport system quadrupoles.

We have, further, succeeded at operating at over $8 \mathrm{~mA}$ by use of the aforementioned skew-quadrupole reflector. When activated, we have verified this system completely cross couples the two transverse planes. Modeling further 
suggests that certain choices of phase advance, in conjunction with the reflector activated, generates a true single turn rotation matrix from the unstable cavity back to itself. We note, however, that this configuration awaits operational evaluation. Though it is possible to manipulate the transfer matrix in this manner, it is not certain that the machine will perform properly, as internal mismatch can generate beam loss that limits machine performance just as surely as BBU.

\section{CONCLUSIONS AND OUTLOOK}

The multipass, multibunch beam breakup instability has been observed at the Jefferson Lab FEL. This has prompted investigations of methods for beam optical control of BBU. From a simple, analytical model and from twodimensional BBU simulations, it was shown that a $90^{\circ}$ rotation of the betatron planes is the most robust and effective means to suppress the instability in a two-pass recirculator using beam optical methods. Experimentally, the skew-quadrupole reflector has been successfully commissioned in the FEL upgrade driver. A systematic and quantitative study of its effect on the threshold current has yet to be performed. However, operational experience has shown that the threshold is increased from $5 \mathrm{~mA}$ to over $8 \mathrm{~mA}$ with the reflector activated without any evidence of BBU induced machine trips.

The FEL is the only currently operating accelerator to observe this particular form of BBU. This gives us the unique opportunity to experimentally study not only the instability itself, but methods of suppression. Experimental studies presented in this paper have been in the regime where the operating current is above the threshold current. Future efforts are focused on developing measurements to determine the threshold current for a particular machine setup while working in the regime where the machine current is below the threshold current.

\section{ACKNOWLEDGMENTS}

This work supported by The Office of Naval Research, the Joint Technology Office, NAVSEA PMS-405, the Air Force Research Laboratory, U.S. Army Night Vision Lab, the Commonwealth of Virginia, and by DOE Contract No. DE-AC05-84ER40150.

\section{APPENDIX: DERIVATION OF $M^{*}$ IN THE THRESHOLD CURRENT FORMULA}

To include the more general case of an HOM with arbitrary polarization and a generalized $4 \times 4$ transport matrix into the analytic model of BBU, one needs to recognize that only the component of the beam displacement on the second pass through the cavity in the direction of the cavity HOM voltage can exchange energy with the mode. Thus, to find the projection of the beam displacement on the HOM voltage we take the following dot product; $r \cdot \hat{r}_{o}^{\prime}$ where $r$ is the displacement on the second pass and $\hat{r}_{o}^{\prime}$ is a unit vector describing the orientation of the HOM. For the case of an HOM oriented with an angle, $\alpha$, with respect to the $x$ axis and an arbitrary $4 \times 4$ recirculation matrix, the transverse deflection caused by the HOM on the first pass is expressed as

$$
r_{o}^{\prime}=\left(x_{o}^{\prime}, y_{o}^{\prime}\right)=\frac{V_{\mathrm{HOM}}}{p_{b}(c / e)}(\cos \alpha, \sin \alpha)
$$

where $V_{\mathrm{HOM}}$ is the deflecting voltage of the HOM experienced by the beam and where $p_{b}$ is the beam momentum at the cavity. The displacement on the second pass can be expressed in terms of the angular deflections received on the first pass and on the recirculation matrix as

$$
r=(x, y)=\left(M_{12} x_{o}^{\prime}+M_{14} y_{o}^{\prime}, M_{32} x_{o}^{\prime}+M_{34} y_{o}^{\prime}\right) .
$$

Performing the requisite dot product yields the following equation:

$$
\begin{aligned}
r \cdot \hat{r}_{o}^{\prime}= & r \cdot \frac{r_{o}^{\prime}}{\left|r_{o}^{\prime}\right|} \\
= & \frac{V_{\mathrm{HOM}}}{p_{b}(c / e)}\left(M_{12} \cos ^{2} \alpha+\left(M_{14}+M_{32}\right) \sin \alpha \cos \alpha\right. \\
& \left.+M_{34} \sin ^{2} \alpha\right) .
\end{aligned}
$$

The above expression describes the transverse displacement on the second pass arising from an initial deflection on the first pass from an arbitrarily polarized HOM with a generalized $4 \times 4$ recirculation transfer map. Recall that in Eq. (1) we defined $M^{*}$ as the quantity in parentheses on the right hand side of Eq. (A3) and which describes how a deflection on the first pass is mapped to a displacement on the second pass.

[1] C. Tennant, K. Jordan, E. Pozdeyev, R. Rimmer, H. Wang, and S. Simrock, in Proceedings of the International Linac Conference, Lubeck, Germany, 2004, pp. 387-389.

[2] R.E. Rand and T. I. Smith, Part. Accel. 11, 1 (1980).

[3] S. Benson, D. Douglas, M. Shinn, K. Beard, C. Behre, G. Biallas, J. Boyce, H.F. Dylla, R. Evans, A. Grippo, J. Gubeli, D. Hardy, C. Hernandez-Garcia, K. Jordan, L. Merminga, G. R. Neil, J. Preble, T. Siggins, R. Walker, G. P. Williams, B. Yunn, S. Zhang, and H. Toyokawa, in Proceedings of the 26th International FEL Conference, Trieste, Italy, 2004, pp. 229-232.

[4] E. Pozdeyev, Phys. Rev. ST Accel. Beams 8, 054401 (2005).

[5] E. Pozdeyev and C. Tennant, JLAB Technical Report No. TN-04-019, 2004.

[6] G. H. Hoffstaetter and I. V. Bazarov, Phys. Rev. ST Accel. Beams 7, 054401 (2004).

[7] K. Beard, JLAB Technical Report No. TN-02-044, 2002.

[8] B. Yunn, in Proceedings of the Particle Accelerator Conference, San Francisco, CA, 1991 (IEEE, Piscataway, NJ, 1991), pp. 1785-1787. 
[9] G. A. Krafft and J. J. Bisognano, in Proceedings of the Particle Accelerator Conference, Washington, DC, 1987 (IEEE, Piscataway, NJ, 1987), pp. 1356-1358.

[10] K. Beard, JLAB Technical Report No. TN-02-045, 2002.

[11] H. Wang, I. Campisi, K. Beard, R. Rimmer, C. Thomas, J. Mammosser, and J. Preble, in Proceedings of the Particle Accelerator Conference, Portland, OR, 2003 (IEEE, Piscataway, NJ, 2003), pp. 1104-1106.

[12] R. Servranckx, K. Brown, L. Schachinger, and D. Douglas, SLAC Report No. SLAC-R-285, 1990.
[13] C. Tennant, E. Pozdeyev, S. Simrock, A. Sun, and H. Wang, JLAB Technical Report No. TN-04-008, 2004.

[14] C. Tennant and E. Pozdeyev, JLAB Technical Report No. TN-04-020, 2004.

[15] D. Douglas, JLAB Technical Report No. TN-04-016, 2004.

[16] D. Douglas, JLAB Technical Report No. TN-04-025, 2004. 